\title{
Energy-Efficient Resource Allocation in a Multi-UAV-Aided NOMA Network
}

\author{
Xing $\mathrm{Xi}^{*}$, Xianbin $\mathrm{Cao}^{*}$, Peng Yang ${ }^{\dagger}$, Jingxuan Chen*, and Dapeng $\mathrm{Wu}^{\ddagger}$ \\ *Beihang University, Beijing, China, email: \{xixing,xbcao,chenjingxuan $\} @$ buaa.edu.cn \\ ${ }^{\dagger}$ Singapore University of Technology and Design, Singapore, email: peng_yang@sutd.edu.sg \\ ${ }_{\ddagger}^{\ddagger}$ University of Florida, Gainesville, FL 32611, USA, email: dpwu@ufl.edu
}

\begin{abstract}
This paper is concerned with the resource allocation in a multi-unmanned aerial vehicle (UAV)-aided network for providing enhanced mobile broadband (eMBB) services for user equipments. Different from most of the existing network resource allocation approaches, we investigate a joint non-orthogonal user association, subchannel allocation and power control problem. The objective of the problem is to maximize the network energy efficiency under the constraints on user equipments' quality of service, UAVs' network capacity and power consumption. We formulate the energy efficiency maximization problem as a challenging mixed-integer non-convex programming problem. To alleviate this problem, we first decompose the original problem into two subproblems, namely, an integer non-linear user association and subchannel allocation subproblem and a non-convex power control subproblem. We then design a twostage approximation strategy to handle the non-linearity of the user association and subchannel allocation subproblem and exploit a successive convex approximation approach to tackle the non-convexity of the power control subproblem. Based on the derived results, we develop an iterative algorithm with provable convergence to mitigate the original problem. Simulation results show that our proposed framework can improve energy efficiency compared with several benchmark algorithms.
\end{abstract}

\section{INTRODUCTION}

Enhanced mobile broadband (eMBB) has been identified as one of the three major services of $5 \mathrm{G}$ wireless networks [1]. To provide high-quality eMBB services, which have high transmission rate requirements, the network capacity of the infrastructure should be robust. However, when network congestion or network failure caused by flash crowd traffic or infrastructure malfunction occurs in an area, terrestrial eMBB users may suffer from communication service interruption.

A promising solution to alleviate the effect of network congestion or network failure is the utilization of unmanned aerial vehicle (UAV) base stations (i.e., low-altitude UAVs equipped with transceivers), which can support fast communication service recovery or even network performance enhancement [2]. Motivated by these advantages, UAV-aided communications are gradually attracting the attention of researchers.

Recent works on the UAV-aided communications mainly focus on network resource allocation. For example, Zhang et al. considered a multi-UAV-aided network and studied a joint subchannel allocation and UAV speed optimization problem to improve the uplink sum rate of the network [3]. Cui et al. investigated a dynamic resource allocation problem of a multiUAV network to maximize long-term rewards. They proposed a multi-agent reinforcement learning-based algorithm to find the optimal strategy on joint user, subchannel and power level selection [4].

However, the above works [3], [4] are all based on orthogonal multiple access (OMA) techniques. To further improve the utilization efficiency of network resources, nonorthogonal multiple access (NOMA) techniques have been studied for the UAV-aided communications. For example, Zhao et al. investigated a joint user scheduling, UAV trajectory and NOMA precoding problem for a UAV-aided NOMA network to maximize users' sum rate [5]. Tang et al. studied a joint placement design, admission control, and power allocation problem for a heavy-loaded UAV-aided NOMA network to maximize the number of served users [6]. Nevertheless, the works in [5], [6] considered single-UAV communications, which have the disadvantages of limited service capability and poor robustness compared to multi-UAV communications. As a result, Duan et al. considered resource allocation for a multi-UAV-aided NOMA uplink network and jointly optimized subchannel allocation, transmit power, and UAVs' heights to improve the system capacity [7]. However, they adopted the classic K-mean clustering method to associate UAVs and users, which had low resource utilization. Meanwhile, the classic K-mean clustering method could not achieve load balance among UAVs. Compared with terrestrial networks, the capacity of UAV networks is stringently limited. Therefore, the proposed resource allocation algorithm in [7] might lead to UAV network congestion.

To improve the resource utilization and achieve load balance in a multi-UAV-aided NOMA downlink network, we investigate a joint non-orthogonal user association, subchannel allocation and power control problem in this paper. The main contributions are summarized as follows:

- We formulate a joint non-orthogonal resource allocation optimization problem aiming at maximizing the network energy efficiency under the constraints on quality of service $(\mathrm{QoS})$ requirements, network capacity, and power consumption.

- The formulated problem is confirmed to be a challenging mixed-integer non-convex programming problem. To alleviate this problem, we decompose it into two separated subproblems, namely, an integer non-linear user association and subchannel allocation subproblem, and a non-convex power control subproblem.

- We then design a two-stage approximation strategy to 
handle the non-linearity of the user association and subchannel allocation subproblem and exploit a successive convex approximation (SCA) approach to tackle the nonconvexity of the power control subproblem. Then an iterative algorithm with provable convergence is proposed to alternatively optimize the above two subproblems.

The rest of this paper is organized as follows: We present the system model and the problem formulation in Section II. We develop the problem solution for the formulated problem in Section III. Section IV shows our simulation results and Section $\mathrm{V}$ concludes this paper.

\section{System Model And Problem Formulation}

\section{A. System Model}

In this paper, we consider a NOMA-based downlink communication scenario. In this scenario, multiple UAV base stations (UBSs) are deployed to assist a macro base station (MBS) to provide eMBB services for a collection of congested terrestrial user equipments (UEs) which cannot be served by the MBS in a geographical area. Denote the set of UBSs and the set of UEs by $\mathcal{J}=\left\{1,2, \ldots, N_{d}\right\}$ and $\mathcal{I}=\left\{1,2, \ldots, N_{u}\right\}$ respectively. We consider that the locations of all UBSs and UEs are fixed and known, and all UBSs are deployed at the same altitude $H$. For simplicity, we ignore the height of the MBS and the UEs. Meanwhile, this paper considers a frequency division multiple access (FDMA) communication system. The total channel bandwidth is $W$ and is equally divided into $N_{s}$ orthogonal subchannels, denoted by $\mathcal{N}=$ $\left\{1,2, \ldots, N_{s}\right\}$. For convenience of description, we denote the subchannel $n$ of UBS $j$ as $\mathcal{S C}_{j n}$. Let $a_{i j n}$ be a binary variable indicating user association and subchannel allocation and let $\mathcal{A}=\left\{a_{i j n}, \forall i, j, n\right\}$ denote the user association and subchannel allocation matrix. We set $a_{i j n}=1$ if the subchannel $\mathcal{S C}_{j n}$ is allocated to UE $i$; otherwise, $a_{i j n}=0$. This paper investigates the optimization of joint user association, subchannel allocation and UBSs' transmit power control, and we assume that the transmit power of the MBS is fixed and known.

Denote the horizontal location of UBS $j$ and the location of UE $i$ by $\boldsymbol{x}_{j}^{d}$ and $\boldsymbol{x}_{i}^{u}$ respectively. This paper leverages the airto-ground (ATG) propagation model [8] to obtain the channel gain from UBS $j$ to UE $i$ on the subchannel $n$, denoted by $h_{i j n}$. For the ATG link, each UE has a line-of-sight (LoS) connection with a UBS with a specific probability. The LoS probability relies on the environment (e.g., rural, suburban, urban and dense urban), the locations of the UBS and the UE, and can be expressed as

$$
P_{L o S}\left(H, d_{i j}^{h}\right)=\frac{1}{1+\alpha_{1} \exp \left(-\alpha_{2}\left(\theta_{i j}-\alpha_{1}\right)\right)},
$$

where $\alpha_{1}$ and $\alpha_{2}$ are constant values depending on the environment, $\theta_{i j}=\frac{180}{\pi} \times \arctan \left(\frac{H}{d_{i j}^{h}}\right)$ is the elevation angle of UE $i$ towards UBS $j$, and $d_{i j}^{h}$ is the horizontal distance between UBS $j$ and UE $i$, i.e., $d_{i j}^{h}=\left\|\boldsymbol{x}_{i}^{u}-\boldsymbol{x}_{j}^{d}\right\|_{2}$. Also, the non-line-of-sight $(\mathrm{NLoS})$ probability is $P_{N L o S}\left(H, d_{i j}^{h}\right)=$
$1-P_{L o S}\left(H, d_{i j}^{h}\right)$. Thus, the channel gain from UBS $j$ to UE $i$ on the subchannel $n$ is

$$
h_{i j n}=\frac{g_{i j n}^{T x} g_{i j n}^{R x} \varsigma^{2}}{16 \pi^{2}\left(\frac{d_{i j}}{d_{0}}\right)^{2}} 10^{-\frac{P_{L o S}\left(H, d_{i j}^{h}\right) \eta_{L o S}^{d B}+P_{N L o S}\left(H, d_{i j}^{h}\right) \eta_{N L o S}^{d B}}{10}},
$$

where $g_{i j n}^{T x}$ and $g_{i j n}^{R x}$ are the transmit and receive antenna gains from UBS $j$ to UE $i$ on the subchannel $n . \varsigma=c / f_{c}$ is the carrier wavelength, where $c$ is the speed of light and $f_{c}$ is the carrier frequency. $d_{i j}=\sqrt{\left(d_{i j}^{h}\right)^{2}+H^{2}}$ is the distance between UBS $j$ and UE $i$ and $d_{0}$ is a far field reference distance. $\eta_{L o S}^{d B}($ in $\mathrm{dB})$ and $\eta_{N L o S}^{d B}($ in $\mathrm{dB}$ ) represent the excessive propagation losses corresponding to the LoS and NLoS connections respectively, which depend on the environment.

Denote the location of the MBS by $\boldsymbol{x}^{M}$. This paper leverages the propagation path loss model [9] to obtain the channel gain from the MBS to UE $i$ on the subchannel $n$, denoted by $h_{i n}^{M}$. Thus, the channel gain $h_{i n}^{M}$ is

$$
h_{i n}^{M}=\frac{g_{i n}^{M T x} g_{i n}^{M R x} \varsigma^{2}}{16 \pi^{2}\left(\frac{d_{i}^{M}}{d_{0}}\right)^{\eta}},
$$

where $g_{i n}^{M T x}$ and $g_{i n}^{M R x}$ are the transmit and receive antenna gains from the MBS to UE $i$ on the subchannel $n, d_{i}^{M}=$ $\left\|\boldsymbol{x}_{i}^{u}-\boldsymbol{x}^{M}\right\|_{2}$ is the distance between the MBS and UE $i$, and $\eta$ is the path loss exponent $(\eta \in[2,6])$.

In the NOMA-based downlink system, the successive interference cancellation (SIC) technique is adopted at the receiver to eliminate the interference from other UEs served by the same subchannel $\mathcal{S C}_{j n}$ in a certain decoding order [5], [10]. We assume that the UE with higher channel gain can decode the signals of the other UEs with worse channel gain served by the same subchannel $\mathcal{S C}_{j n}$, and the transmit power allocated to the former is not more than that of the latter. Owing to the high implementation complexity and decoding complexity of SIC and the high complexity of resource allocation algorithms, like [11], we investigate the case that each $\mathcal{S C}_{j n}$ can be allocated to at most two UEs. In consequence, we have

$$
\begin{gathered}
\mathrm{C} 1: \sum_{i \in \mathcal{I}} a_{i j n} \leq 2, \forall j \in \mathcal{J}, n \in \mathcal{N}, \\
\mathrm{C} 2: a_{i j n} \in\{0,1\}, \forall i \in \mathcal{I}, j \in \mathcal{J}, n \in \mathcal{N} .
\end{gathered}
$$

Considering the number of UEs served by the subchannel $\mathcal{S C}_{j n}$, we calculate the received signal-to-interference-plusnoise ratio (SINR) in the following two cases.

Case 1: When $\mathcal{S C}_{j n}$ is allocated to only one UE $i$, we name $\mathrm{UE} i$ as a primary $\mathrm{UE}$ on $\mathcal{S C}_{j n}$. Then, the received SINR of the primary $\mathrm{UE} i$ on $\mathcal{S C}_{j n}$ is

$$
\gamma_{i j n}=\frac{p_{1, j n} h_{i j n}}{\sum_{k \neq j, k \in \mathcal{J}} p_{k n} h_{i k n}+p_{n}^{M} h_{i n}^{M}+\sigma_{n}^{2}} .
$$

Case 2: When $\mathcal{S C}_{j n}$ is allocated to two UEs $i_{1}$ and $i_{2}$ with $h_{i_{1} j n}>h_{i_{2} j n}$, i.e., UE $i_{1}$ can eliminate the interference of UE $i_{2}$ on $\mathcal{S C}_{j n}$, we name UE $i_{1}$ and UE $i_{2}$ as a primary UE and a 
secondary UE on $\mathcal{S C}_{j n}$ respectively. Then, the received SINRs of the primary UE $i_{1}$ and the secondary $\mathrm{UE} i_{2}$ on $\mathcal{S C}_{j n}$ are

$$
\begin{gathered}
\gamma_{i_{1} j n}=\frac{p_{1, j n} h_{i_{1} j n}}{\sum_{k \neq j, k \in \mathcal{J}} p_{k n} h_{i_{1} k n}+p_{n}^{M} h_{i_{1} n}^{M}+\sigma_{n}^{2}}, \\
\gamma_{i_{2} j n}=\frac{p_{2, j n} h_{i_{2} j n}}{p_{1, j n} h_{i_{2} j n}+\sum_{k \neq j, k \in \mathcal{J}} p_{k n} h_{i_{2} k n}+p_{n}^{M} h_{i_{2} n}^{M}+\sigma_{n}^{2}},
\end{gathered}
$$

where $p_{1, j n}$ and $p_{2, j n}$ are the transmit powers allocated to the primary UE and the secondary $\mathrm{UE}$ on $\mathcal{S C}_{j n}$ respectively, $p_{j n}=p_{1, j n}+p_{2, j n}$ is the total transmit power on $\mathcal{S C}_{j n}, p_{n}^{M}$ is the transmit power of the MBS on the subchannel $n$, and $\sigma_{n}^{2}$ represents the additive white Gaussian noise on the subchannel $n$. Let $\mathcal{S}_{c 2}=\left\{(j, n) \mid \sum_{i \in \mathcal{I}} a_{i j n}=2\right\}$ represent the index set corresponding to the case 2 . Referring to the definition of $p_{1, j n}$ and $p_{2, j n}$ and the assumption of SIC, $p_{1, j n}$ and $p_{2, j n}$ satisfy the following constraints

$$
\begin{gathered}
\text { C3: } p_{2, j n}=0, \forall(j, n) \notin \mathcal{S}_{c 2}, \\
\text { C4: } p_{1, j n} \leq p_{2, j n}, \forall(j, n) \in \mathcal{S}_{c 2} .
\end{gathered}
$$

Let $\mathcal{P}=\left\{p_{1, j n}, p_{2, j n}, \forall j, n\right\}$ denote the transmit power matrix. According to the Shannon capacity, the achievable data transfer rate of $\mathrm{UE} i$ on $\mathcal{S C}_{j n}$ is

$$
r_{i j n}=\frac{W}{N_{s}} \log _{2}\left(1+\gamma_{i j n}\right) .
$$

We define UEs' different QoS requirements by their achievable data transfer rate $(\mathrm{Mb} / \mathrm{s})$. Denote the achievable data transfer rate and the minimum required data transfer rate of $\mathrm{UE} i$ as $R_{i}$ and $R_{i}^{\min }$ respectively. Thus, we have

$$
\text { C5: } R_{i}=\sum_{j \in \mathcal{J}} \sum_{n \in \mathcal{N}} a_{i j n} r_{i j n} \geq R_{i}^{\min }, \forall i \in \mathcal{I} \text {. }
$$

Besides, UBS $j$ needs to receive data that will be forwarded to UEs from a ground station via an uplink with limited network capacity $(\mathrm{Mb} / \mathrm{s})$. In this paper, we regard it as the maximum network capacity of UBS $j$, denoted by $C_{j}^{\max }$. Thus, we have

$$
\text { C6: } \sum_{i \in \mathcal{I}} \sum_{n \in \mathcal{N}} a_{i j n} r_{i j n} \leq C_{j}^{\max }, \forall j \in \mathcal{J} .
$$

Next, let $p_{j}, p_{j}^{c}$ and $p_{j}^{\max }$ denote the transmit power, the circuit power and the maximum power consumption limit of UBS $j$. Thus, we have

$$
\begin{gathered}
\text { C7: } p_{j}=\sum_{n \in \mathcal{N}}\left(p_{1, j n}+p_{2, j n}\right), \forall j \in \mathcal{J}, \\
\text { C8: } p_{j}+p_{j}^{c} \leq p_{j}^{\max }, \forall j \in \mathcal{J}, \\
\text { C9: } p_{1, j n} \geq 0, p_{2, j n} \geq 0, \forall j \in \mathcal{J}, n \in \mathcal{N} .
\end{gathered}
$$

We denote the energy efficiency as $f_{E E}$. Considering the fairness of service among UEs and the fairness of power consumption among UBSs, we define $f_{E E}$ as the ratio of the product of the number of UEs and the minimum achievable data transfer rate among all UEs and the product of the number of UBSs and the maximum power consumption among all UBSs. As such, the objective function can be written as

$$
f_{E E}=\frac{N_{u} \cdot \min _{i \in \mathcal{I}} R_{i}}{N_{d} \cdot \max _{j \in \mathcal{J}}\left(p_{j}+p_{j}^{c}\right)},
$$

where $N_{u} \cdot \min _{i \in \mathcal{I}} R_{i}$ represents the lower bound of the total achievable data transfer rate of all UEs and $N_{d} \cdot \max _{j \in \mathcal{J}}\left(p_{j}+p_{j}^{c}\right)$ represents the upper bound of the total power consumption of all UBSs.

\section{B. Problem Formulation}

Considering all constraints and the objective function mentioned above, we can formulate the joint association, subchannel and power optimization problem as

$$
\begin{aligned}
& \max _{\mathcal{A}, \mathcal{P}} f_{E E}=\frac{N_{u}}{N_{d}} \cdot \frac{\min _{i \in \mathcal{I}} R_{i}}{\max _{j \in \mathcal{J}}\left(p_{j}+p_{j}^{c}\right)} \\
& \text { s.t. (4), (5), (9), (10), (12), (13), (14), (15), (16). }
\end{aligned}
$$

Since both $N_{u}$ and $N_{d}$ are given constants, solving (18) is equivalent to the solution of the following (19)

$$
\begin{aligned}
& \max _{\mathcal{A}, \mathcal{P}} \eta_{E E}=\frac{\min _{i \in \mathcal{I}} R_{i}}{\max _{j \in \mathcal{J}}\left(p_{j}+p_{j}^{c}\right)} \\
& \text { s.t. (4), (5), (9), (10), (12), (13), (14), (15), (16). }
\end{aligned}
$$

We define the optimal $\eta_{E E}^{*}$ as

$$
\eta_{E E}^{*}=\frac{\min _{i \in \mathcal{I}} R_{i}\left(\mathcal{A}^{*}, \mathcal{P}^{*}\right)}{\max _{j \in \mathcal{J}}\left(p_{j}\left(\mathcal{P}^{*}\right)+p_{j}^{c}\right)},
$$

where $\mathcal{A}^{*}$ and $\mathcal{P}^{*}$ denote the optimal user association and subchannel allocation and the optimal transmit power when yielding $\eta_{E E}^{*}$.

Lemma 1. $\eta_{E E}^{*}$ can be achieved if and only if [10]

$$
\begin{aligned}
& \max _{\mathcal{A}, \mathcal{P}}\left(\min _{i \in \mathcal{I}} R_{i}(\mathcal{A}, \mathcal{P})\right)-\eta_{E E}^{*}\left(\max _{j \in \mathcal{J}}\left(p_{j}(\mathcal{P})+p_{j}^{c}\right)\right) \\
& =\left(\min _{i \in \mathcal{I}} R_{i}\left(\mathcal{A}^{*}, \mathcal{P}^{*}\right)\right)-\eta_{E E}^{*}\left(\max _{j \in \mathcal{J}}\left(p_{j}\left(\mathcal{P}^{*}\right)+p_{j}^{c}\right)\right)=0 .
\end{aligned}
$$

Proof. A similar proof can be found in [10].

According to Lemma 1, we can transform the objective function in (19) into a subtractive form, and the problem (19) can be rewritten as

$$
\begin{aligned}
& \max _{\mathcal{A}, \mathcal{P}}\left(\min _{i \in \mathcal{I}} R_{i}\right)-\eta_{E E}\left(\max _{j \in \mathcal{J}}\left(p_{j}+p_{j}^{c}\right)\right) \\
& \text { s.t. (4), (5), (9), (10), (12), (13), (14), (15), (16). }
\end{aligned}
$$

In the problem (22), (4), (5), (12) and (13) involve binary variables $\left\{a_{i j n}\right\}$. Further, even if $\left\{a_{i j n}\right\}$ are fixed, (12) and (13) are not convex constraints. Therefore, (22) is a mixedinteger non-convex programming problem, which is indeterminable or NP-hard [12] and challenging to be solved. Besides, $\mathcal{A}$ and $\mathcal{P}$ are coupled in the objective function and the constraints (12) and (13), which increases the difficulty of mitigating (22). Fortunately, we observe that the complexity of (22) may be weakened if $\mathcal{A}$ and $\mathcal{P}$ can be decoupled. Based on this crucial observation, we first decompose (22) into two separated subproblems, namely, association and subchannel optimization with fixed transmit power and power optimization 
with fixed user association and subchannel allocation. Based on the solutions of the above two subproblems, we then develop an iterative algorithm for (22) to alternatively optimize these two subproblems. The detailed procedures are described in the following section.

\section{PROBLEM SOLUTION}

\section{A. User Association and Subchannel Allocation}

For any given transmit power $\mathcal{P}$, this subsection considers the subproblem of (22) of user association and subchannel allocation. By introducing auxiliary variables $\eta_{R}$ and $\left\{\eta_{i}, \forall i \in\right.$ $\mathcal{I}$ \}, the user association and subchannel allocation subproblem can be formulated as

$$
\begin{aligned}
& \max _{\mathcal{A}, \eta_{R},\left\{\eta_{i}\right\}} \eta_{R} \\
& \text { s.t. } \\
& \eta_{i}=\sum_{j \in \mathcal{J}} \sum_{n \in \mathcal{N}} a_{i j n} r_{i j n}(\mathcal{A}), \forall i \in \mathcal{I}, \\
& \eta_{i} \geq R_{i}^{\min }, \forall i \in \mathcal{I}, \\
& \eta_{i} \geq \eta_{R}, \forall i \in \mathcal{I},
\end{aligned}
$$$$
\text { (4), (5) and (13) are satisfied, }
$$

where $\eta_{i}$ represents the achievable data transfer rate of UE $i$ and $\eta_{R}$ represents the minimum achievable data transfer rate among all UEs.

However, the above problem (23) is challenging to be solved since the achievable data transfer rate $r_{i j n}(\mathcal{A})$ is not a fixed value even with fixed transmit power $\mathcal{P}$. From (6), (7), (8) and (11), it can be observed that $r_{i j n}(\mathcal{A})$ takes different values depending on whether UE $i$ is a primary $\mathrm{UE}$ or a secondary UE on $\mathcal{S C}_{j n}$. Therefore, the value of $r_{i j n}(\mathcal{A})$ depends on $\mathcal{A}$ with the fixed $\mathcal{P}$. According to this key observation, we propose a two-stage approximation strategy to further decompose the problem (23) into two integer linear programming (ILP) problems, both of which can be solved efficiently by existing optimization tools such as MOSEK [13]. The detailed procedures are described as follows.

1) Primary User Association and Subchannel Allocation: We assume that each $\mathcal{S C}_{j n}$ can be allocated to at most one UE at the primary user association and subchannel allocation stage (hereinafter referred to as the primary stage). Therefore, each UE $i$ can be regarded as a primary UE on $\mathcal{S C}_{j n}$ at this stage, and thus we can formulate the primary user association and subchannel allocation subproblem as the following ILP problem

$$
\begin{aligned}
& \max _{\mathcal{A}, \eta_{R},\left\{\eta_{i}\right\}} \eta_{R} \\
& s . t \\
& \sum_{i \in \mathcal{I}} a_{i j n} \leq 1, \forall j \in \mathcal{J}, n \in \mathcal{N}, \\
& \eta_{i}=\sum_{j \in \mathcal{J}} \sum_{n \in \mathcal{N}} a_{i j n} r_{i j n}^{p}, \forall i \in \mathcal{I}, \\
& \eta_{i} \geq R_{i}^{\min }, \forall i \in \mathcal{I}, \\
& \eta_{i} \geq \eta_{R}, \forall i \in \mathcal{I}, \\
& \sum_{i \in \mathcal{I}} \sum_{n \in \mathcal{N}} a_{i j n} r_{i j n}^{p} \leq C_{j}^{\max }, \forall j \in \mathcal{J}, \\
& a_{i j n} \in\{0,1\}, \forall i \in \mathcal{I}, j \in \mathcal{J}, n \in \mathcal{N},
\end{aligned}
$$

where $r_{i j n}^{p}=\frac{W}{N_{s}} \log _{2}\left(1+\frac{p_{1, j n} h_{i j n}}{\sum_{k \neq j, j \in \mathcal{J}} p_{k n} h_{i k n}+p_{n}^{M} h_{i n}^{M}+\sigma_{n}^{2}}\right)$.
Let $\left\{a_{i j n}^{p *}\right\}$ denote the solution of (24) and $\mathcal{S}_{p *}=$ $\left\{(i, j, n) \mid a_{i j n}^{p *}=1\right\}$ denote the index set of the user association and subchannel allocation determined at the primary stage. In addition, for the convenience of the description of the following secondary user association and subchannel allocation, we let $R_{i}^{p}=\sum_{j \in \mathcal{J}} \sum_{n \in \mathcal{N}} a_{i j n}^{p *} r_{i j n}^{p}$ and $C_{j}^{p}=\sum_{i \in \mathcal{I}} \sum_{n \in \mathcal{N}} a_{i j n}^{p *} r_{i j n}^{p}$ represent the achievable data transfer rate of UE $i$ and the total data transfer rate of UBS $j$ at the primary stage respectively.

2) Secondary User Association and Subchannel Allocation: Similarly, we assume that each $\mathcal{S C}_{j n}$ can be allocated to at most one UE at the secondary user association and subchannel allocation stage (hereinafter referred to as the secondary stage). Particularly, based on the primary stage, we can calculate the achievable data transfer rate $r_{i j n}$ at the secondary stage in the following two cases.

Case 1: For each $\mathcal{S C}_{j n}$, if $\mathcal{S C}_{j n}$ is not allocated to any UE at the primary stage, then UE $i$ is a primary UE on $\mathcal{S C}_{j n}$ at the secondary stage. Let $\mathcal{S}_{u}=\left\{(i, j, n) \mid \sum_{k \in \mathcal{I}} a_{k j n}^{p *}=0\right\}$ represent the index set of the user association and subchannel allocation corresponding to this case. Thus, for each $(i, j, n) \in$ $\mathcal{S}_{u}$, the achievable data transfer rate $r_{i j n}^{u}$ at the secondary stage is

$$
r_{i j n}^{u}=\frac{W}{N_{s}} \log _{2}\left(1+\frac{p_{1, j n} h_{i j n}}{\sum_{k \neq j, k \in \mathcal{J}} p_{k n} h_{i k n}+p_{n}^{M} h_{i n}^{M}+\sigma_{n}^{2}}\right) .
$$

Case 2: If $\mathcal{S C}_{j n}$ is allocated to a UE at the primary stage, then we denote this UE as $i_{p}$, i.e., $\left(i_{p}, j, n\right) \in \mathcal{S}_{p *}$. When $\mathcal{S C}_{j n}$ is allocated to UE $i_{p}$ and UE $i$ at the primary and secondary stages respectively, one of the two UEs is a primary UE and the other is a secondary UE on $\mathcal{S C}_{j n}$, which is determined by the relative relationship of the two UEs' channel gains. If $h_{i j n}>h_{i_{p} j n}$, then UE $i$ and UE $i_{p}$ are the primary UE and the secondary UE on $\mathcal{S C}_{j n}$ respectively, and the achievable data transfer rate $r_{i_{p} j n}^{p}$ of UE $i_{p}$ at the primary stage will change. Let $\mathcal{S}_{o 1}=\left\{(i, j, n) \mid h_{i j n}>h_{i_{p} j n},\left(i_{p}, j, n\right) \in \mathcal{S}_{p *}\right\}$ represent the index set of the user association and subchannel allocation corresponding to this case. Thus, for each $(i, j, n) \in \mathcal{S}_{o 1}$, the achievable data transfer rate $r_{i j n}^{o 1}$ at the secondary stage is

$$
r_{i j n}^{o 1}=\frac{W}{N_{s}} \log _{2}\left(1+\frac{p_{1, j n} h_{i j n}}{\sum_{k \neq j, k \in \mathcal{J}} p_{k n} h_{i k n}+p_{n}^{M} h_{i n}^{M}+\sigma_{n}^{2}}\right),
$$

and the change of the achievable data transfer rate $r_{i_{p} j n}^{p}$ at the primary stage is

$$
\begin{aligned}
& \Delta r_{i_{p} j n}^{p}= \\
& \frac{W}{N_{s}} \log _{2}\left(1+\frac{p_{2, j n} h_{i_{p} j n}}{p_{1, j n} h_{i_{p} j n}+\sum_{\substack{k \neq j, j \in \mathcal{J} \\
k n n}} p_{k n} h_{i_{p} k n}+p_{n}^{M} h_{i_{p} n}^{M}+\sigma_{n}^{2}}\right) \\
& -\frac{W}{N_{s}} \log _{2}\left(1+\frac{p_{1, j n} h_{i_{p} j n}}{\sum_{k \neq j, j \in \mathcal{J}} p_{k n} h_{i_{p} k n}+p_{n}^{M} h_{i_{p} n}^{M}+\sigma_{n}^{2}}\right) .
\end{aligned}
$$

If $h_{i j n}<h_{i_{p} n}$, then UE $i_{p}$ and UE $i$ are the primary UE and the secondary $\mathrm{UE}$ on $\mathcal{S C}_{j n}$ respectively. Let $\mathcal{S}_{o 2}=\left\{(i, j, n) \mid h_{i j n}<h_{i_{p} j n},\left(i_{p}, j, n\right) \in \mathcal{S}_{p *}\right\}$ represent the index set of the user association and subchannel allocation 
corresponding to this case. Thus, for each $(i, j, n) \in \mathcal{S}_{o 2}$, the achievable data transfer rate $r_{i j n}^{o 2}$ at the secondary stage is

$$
r_{i j n}^{o 2}=\frac{W}{N_{s}} \log _{2}\left(1+\frac{p_{2, j n} h_{i j n}}{p_{1, j n} h_{i j n}+\sum_{k \neq j, j \in \mathcal{J}} p_{k n} h_{i k n}+p_{n}^{M} h_{i n}^{M}+\sigma_{n}^{2}}\right) .
$$

Let $\mathcal{S}_{2}=\mathcal{S}_{u} \cup \mathcal{S}_{o 1} \cup \mathcal{S}_{o 2}$ represent the candidate index set of the feasible user association and subchannel allocation at the secondary stage, and let $\mathcal{S}_{J N}^{p *}(i)=\{(j, n) \mid(i, j, n) \in$ $\left.\mathcal{S}_{p *}\right\}, \mathcal{I}_{o 1}(j, n)=\left\{i \mid(i, j, n) \in \mathcal{S}_{o 1}\right\}$ and $\mathcal{S}_{I N}^{o 1}(j)=$ $\left\{(i, n) \mid(i, j, n) \in \mathcal{S}_{o 1}\right\}$. For each $i \in \mathcal{I}$, the change of the achievable data transfer rate of UE $i$ at the primary stage is

$$
\Delta R_{i}^{p}=\sum_{(j, n) \in \mathcal{S}_{J N}^{p *}(i)} \sum_{k \in \mathcal{I}_{o 1}(j, n)} a_{k j n} \Delta r_{i j n}^{p} .
$$

For each $j \in \mathcal{J}$, the change of the total data transfer rate of UBS $j$ at the primary stage is

$$
\Delta C_{j}^{p}=\sum_{(i, n) \in \mathcal{S}_{I N}^{o 1}(j)} a_{i j n} \Delta r_{i_{p} j n}^{p} .
$$

Based on the above derivations, we can formulate the secondary user association and subchannel allocation subproblem as the following ILP problem

$$
\begin{aligned}
& \max _{\mathcal{A}, \eta_{R},\left\{\eta_{i}\right\}} \eta_{R} \\
& s . t \\
& \sum_{i \in \mathcal{I}} a_{i j n} \leq 1, \forall j \in \mathcal{J}, n \in \mathcal{N}, \\
& \eta_{i}=\sum_{j \in \mathcal{J}} \sum_{n \in \mathcal{N}} a_{i j n} r_{i j n}^{s}+\Delta R_{i}^{p}+R_{i}^{p}, \forall i \in \mathcal{I}, \\
& \eta_{i} \geq R_{i}^{\min }, \forall i \in \mathcal{I}, \\
& \eta_{i} \geq \eta_{R}, \forall i \in \mathcal{I}, \\
& \sum_{i \in \mathcal{I}} \sum_{n \in \mathcal{N}} a_{i j n} r_{i j n}^{s}+\Delta C_{j}^{p}+C_{j}^{p} \leq C_{j}^{\max }, \forall j \in \mathcal{J}, \\
& a_{i j n} \in\{0,1\}, \forall(i, j, n) \in \mathcal{S}_{2}, \\
& a_{i j n}=0, \forall(i, j, n) \notin \mathcal{S}_{2},
\end{aligned}
$$$$
\text { where } r_{i j n}^{s}= \begin{cases}r_{i j n}^{u}, & \forall(i, j, n) \in \mathcal{S}_{u}, \\ r_{i j n}^{o 1}, & \forall(i, j, n) \in \mathcal{S}_{o 1}, \\ r_{i j n}^{o 2}, & \forall(i, j, n) \in \mathcal{S}_{o 2}, \\ 0, & \forall(i, j, n) \notin \mathcal{S}_{2} .\end{cases}
$$

Let $\left\{a_{i j n}^{s *}\right\}$ denote the solution of (31), and then the solution of (23) can be approximated as $\left\{a_{i j n}^{*}\right\}=\left\{a_{i j n}^{p *}+a_{i j n}^{s *}\right\}$.

\section{B. Power Control}

For any given user association and subchannel allocation $\mathcal{A}$, this subsection considers the subproblem of (22) of transmit power control. Let $\mathcal{S}_{a}=\left\{(i, j, n) \mid a_{i j n}=1\right\}$ and $\mathcal{I}_{a}(j, n)=\left\{i \mid a_{i j n}=1\right\}$ represent the index set of the user association and subchannel allocation and the index set of UEs served by $\mathcal{S C}_{j n}$ respectively. Then we divide the user association and subchannel allocation into two categories. One category is that UE $i$ is a secondary $\mathrm{UE}$ on $\mathcal{S C}_{j n}$, and let $\mathcal{S}_{a s}=\left\{(i, j, n) \in \mathcal{S}_{a} \mid \sum_{k \in \mathcal{I}} a_{k j n}=2, h_{i j n}<\max _{k \in \mathcal{I}_{a}(j, n)} h_{k j n}\right\}$ represent the index set of such user association and subchannel allocation. The other category is that UE $i$ is a primary UE on $\mathcal{S C}_{j n}$, and let $\mathcal{S}_{a p}=\mathcal{S}_{a} \backslash \mathcal{S}_{a s}$ represent the index set of such user association and subchannel allocation.
Besides, let $\mathcal{S}_{J N}^{a p}(i)=\left\{(j, n) \mid(i, j, n) \in \mathcal{S}_{a p}\right\}, \mathcal{S}_{J N}^{a s}(i)=$ $\left\{(j, n) \mid(i, j, n) \in \mathcal{S}_{a s}\right\}, \mathcal{S}_{I N}^{a p}(j)=\left\{(i, n) \mid(i, j, n) \in \mathcal{S}_{a p}\right\}$, and $\mathcal{S}_{I N}^{a s}(j)=\left\{(i, n) \mid(i, j, n) \in \mathcal{S}_{a s}\right\}$. Based on the above defined sets and by introducing auxiliary variables $\eta_{R}, \eta_{P}$ and $\left\{\eta_{i}, \forall i \in \mathcal{I}\right\}$, the power control subproblem can be formulated as

$$
\begin{aligned}
& \max _{\mathcal{P}, \eta_{R}, \eta_{P},\left\{\eta_{i}\right\},\left\{p_{j}\right\}} \eta_{R}-\eta_{E E} \eta_{P} \\
& \text { s.t } \\
& \quad \sum_{(j, n) \in \mathcal{S}_{J N}^{a p}(i)} r_{i j n}^{p}(\mathcal{P})+\sum_{(j, n) \in \mathcal{S}_{J N}^{a s}(i)} r_{i j n}^{s}(\mathcal{P}) \geq \eta_{i}, \forall i \in \mathcal{I}, \\
& \eta_{i} \geq R_{i}^{\min }, \forall i \in \mathcal{I}, \\
& \eta_{i} \geq \eta_{R}, \forall i \in \mathcal{I}, \\
& \quad \sum_{(i, n) \in \mathcal{S}_{I N}^{a p}(j)} r_{i j n}^{p}(\mathcal{P})+\sum_{(i, n) \in \mathcal{S}_{I N}^{a s}(j)} r_{i j n}^{s}(\mathcal{P}) \leq C_{j}^{\max }, \forall j \in \mathcal{J}, \\
& p_{j}=\sum_{n \in \mathcal{N}}\left(p_{1, j n}+p_{2, j n}\right), \forall j \in \mathcal{J}, \\
& p_{j}+p_{j}^{c} \leq \eta_{P}, \forall j \in \mathcal{J}, \\
& p_{j}+p_{j}^{c} \leq p_{j}^{\max }, \forall j \in \mathcal{J},
\end{aligned}
$$

(9), (10) and (16) are satisfied.

where $\eta_{i}$ represents the achievable data transfer rate of UE $i, \eta_{R}$ represents the minimum achievable data transfer rate among all UEs and $\eta_{P}$ represents the maximum power consumption among all UBSs.

$$
\begin{aligned}
& r_{i j n}^{p}(\mathcal{P})= \\
& \quad \frac{W}{N_{s}} \log _{2}\left(1+\frac{p_{1, j n} h_{i j n}}{\sum_{k \neq j, k \in \mathcal{J}}\left(p_{1, k n}+p_{2, k n}\right) h_{i k n}+p_{n}^{M} h_{i n}^{M}+\sigma_{n}^{2}}\right), \\
& r_{i j n}^{s}(\mathcal{P})= \\
& \frac{W}{N_{s}} \log _{2}\left(1+\frac{p_{2, j n} h_{i j n}}{p_{1, j n} h_{i j n}+\sum_{k \neq j, k \in \mathcal{J}}\left(p_{1, k n}+p_{2, k n}\right) h_{i k n}+p_{n}^{M} h_{i n}^{M}+\sigma_{n}^{2}}\right) .
\end{aligned}
$$

Note that in $32 \mathrm{~b})$ and $(32 \mathrm{~b}), r_{i j n}^{p}(\mathcal{P})$ and $r_{i j n}^{s}(\mathcal{P})$ are neither convex nor concave with respect to $\mathcal{P}$. Thus, (32 b) and (32) are not convex constraints, and the problem (32) is a nonconvex optimization problem. To solve this non-convex problem, we attempt to approximate the non-convex constraints as convex ones and then transform the non-convex problem into a convex one. To this aim, we resort to a successive convex approximation (SCA) approach [14]. The SCA approach is an efficient technique to solve various types of non-convex optimization problems. The core idea of the SCA approach can be briefly described as approximating the original function as a more tractable function at a given point in each iteration. Specifically, the following lemma shows a method of tackling the non-convex constraints and approximating the non-convex (32) as a convex one. The approximated convex problem can be solved efficiently by existing optimization tools such as MOSEK [13].

Lemma 2. By exploring the SCA approach, 32 can be approximated into a convex optimization problem. Besides, the solution of the approximated problem is feasible for (32). 
Proof. Please refer to Appendix A.

\section{Iterative Association, Subchannel and Power Optimization}

Based on the above derivations, we propose an iterative association, subchannel and power optimization (IASPO) algorithm to solve (22), which is summarized in Algorithm 1 For convenience of description, we let $\eta_{R}=\min _{i \in \mathcal{I}} R_{i}, \eta_{P}=$ $\max _{j \in \mathcal{J}}\left(p_{j}+p_{j}^{c}\right)$. Besides, the following lemma declares the convergence and complexity of the IASPO algorithm.

Lemma 3. The IASPO algorithm is convergent, and its complexity is $O\left(r_{\max }\left(2\left(N_{u}+1\right)^{N_{d} \cdot N_{s}}+\left(2 N_{d} \cdot N_{s}\right)^{3.5}\right)\right)$ in the worst case.

Proof. Please refer to Appendix B.

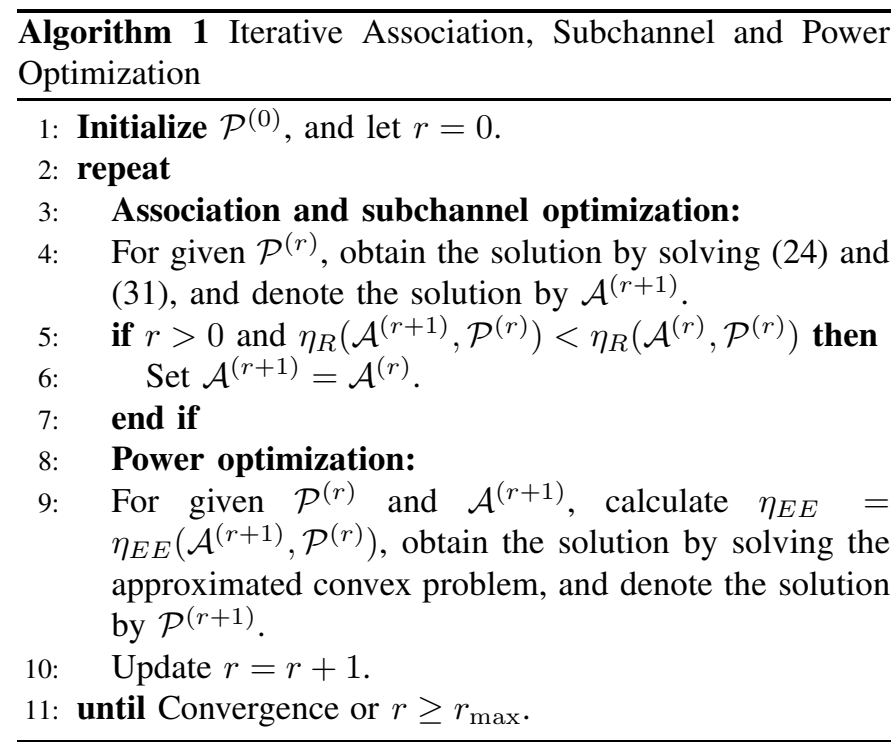

\section{Simulation RESUlts}

\section{A. Comparison Algorithms and Parameter Setting}

To our best knowledge, there are no existing works to be compared. Therefore, to validate the effectiveness of the proposed IASPO algorithm, we compare the proposed algorithm with two benchmark algorithms: 1) Association and subchannel optimization-only (ASOO) algorithm: Allocate transmit power according to the initial power $\mathcal{P}^{(0)}$, and optimize user association and subchannel allocation by solving (24) and (31). 2) IASPO-FDMA algorithm: This algorithm is similar to the IASPO algorithm, except that it considers an FDMA-based communication scenario where each subchannel $\mathcal{S C}_{j n}$ can be allocated to at most one UE.

Set the size of the considered geographic area is a disc of radius $R_{u}=500 \mathrm{~m}$. The MBS is located at the center $(0,0)$, and the UEs and UBSs are uniformly distributed in the annulus $\left(R_{l}, R_{u}\right)$, where $R_{l}=250 \mathrm{~m}$. For each UE $i \in \mathcal{I}$, $R_{i}^{\min }$ is subject to a uniform distribution $U\left(R_{\text {low }}^{\min }, R_{u p}^{\min }\right)$, and $R_{\text {low }}^{\min }=1 \mathrm{Mb} / \mathrm{s}, R_{u p}^{\min }=2 \mathrm{Mb} / \mathrm{s}$. For each UBS $j \in \mathcal{J}$, $p_{j}^{c}=20 \mathrm{dBm}, p_{j}^{\max }=24 \mathrm{dBm}$, and $C_{j}^{\max }=100 \mathrm{Mb} / \mathrm{s}$. More simulation parameters are listed in Table $\llbracket$
TABLE I

SYSTEM PARAMETERS

\begin{tabular}{|c|c|c|c|}
\hline Parameters & Value & Parameters & Value \\
\hline$H$ & $100 \mathrm{~m}$ & $d_{0}$ & 1 \\
\hline$N_{s}$ & 4 & $g_{i j n}^{T x}$ & 1 \\
\hline$W$ & $40 \mathrm{MHz}$ & $g_{i j n}^{R x}$ & 1 \\
\hline$\alpha_{1}$ & 4.88 & $g_{i n}^{M T x}$ & 1 \\
\hline$\alpha_{2}$ & 0.43 & $g_{i n}^{M R x}$ & 1 \\
\hline$p_{n}^{M}$ & $24 \mathrm{dBm}$ & $\eta_{L o S}^{d B}$ & 0.1 \\
\hline$\sigma_{n}^{2}$ & $-85 \mathrm{dBm}$ & $\eta_{N L O S}^{d B}$ & 21 \\
\hline$f_{c}$ & $2.5 \mathrm{GHz}$ & $\eta$ & 3 \\
\hline$c$ & $3 \times 10^{8} \mathrm{~m} / \mathrm{s}$ & $r_{\max }$ & 1000 \\
\hline
\end{tabular}

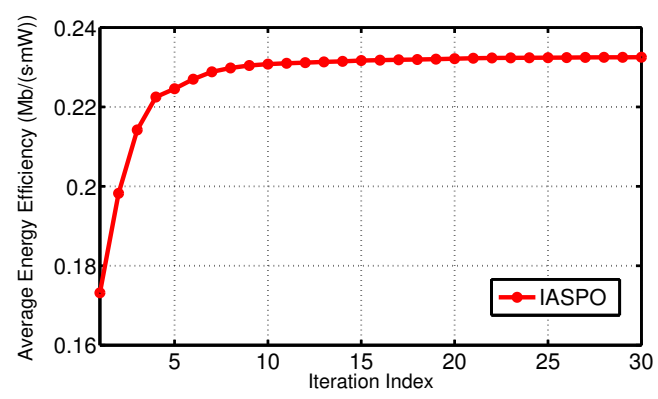

Fig. 1. Average energy efficiency vs. iteration index when $N_{u}=10, N_{d}=4$.

\section{B. Performance Evaluation}

All comparison algorithms need to initialize $\mathcal{P}^{(0)}$. For the algorithms except the IASPO-FDMA algorithm, we initialize $\mathcal{P}^{(0)}$ to $p_{1, j n}^{(0)}=p_{2, j n}^{(0)}=\frac{p_{j}^{\max }-p_{j}^{c}}{4 N_{s}}$ for all $j \in \mathcal{J}, n \in \mathcal{N}$. For the IASPO-FDMA algorithm, we initialize $\mathcal{P}^{(0)}$ to $p_{j n}^{(0)}=$ $\frac{p_{j}^{\max }-p_{j}^{c}}{2 N_{s}}$ for all $j \in \mathcal{J}, n \in \mathcal{N}$.

We perform all comparison algorithms on one hundred randomly generated data sets in the simulation, and the final result is the average of the one hundred results.

We first study the convergence of the proposed IASPO algorithm. Fig. 11 illustrates the convergence behaviour of the energy efficiency $f_{E E}$ of the IASPO algorithm. We can observe that $f_{E E}$ increases monotonously with the increase of the iteration index and quickly converges to a certain value.

Then we consider the effect of the number of UEs $N_{u}$ and the number of UBSs $N_{d}$ on the energy efficiency $f_{E E}$ for all comparison algorithms. Fig. 2 illustrates the energy efficiency vs. the number of UEs and Fig. 3 illustrates the energy efficiency vs. the number of UBSs. From Figs. 2, 3, we can observe that:

- The IASPO algorithm can achieve the highest energy efficiency compared with the other two algorithms except when $N_{u}=6$ and $N_{d}=4$. Given the number of UAVs (e.g., $N_{d}=4$ ), when the number of UEs is greater than six, the IASPO algorithm outperforms the IASPOFDMA algorithm. When the number of UEs is six, the performance of the IASPO-FDMA algorithm is better than that of the the IASPO algorithm. This is because 


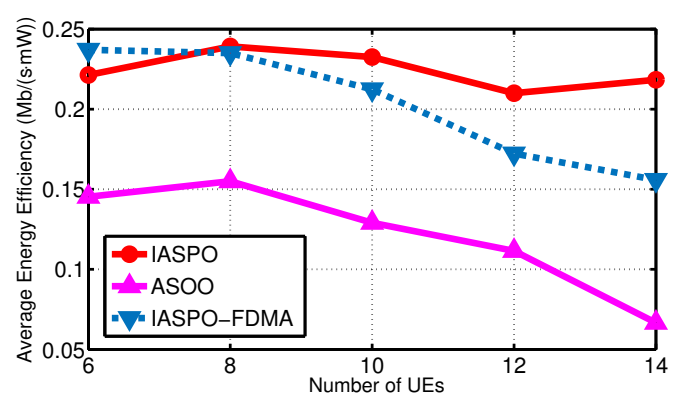

Fig. 2. Average energy efficiency vs. the number of UEs when $N_{d}=4$.

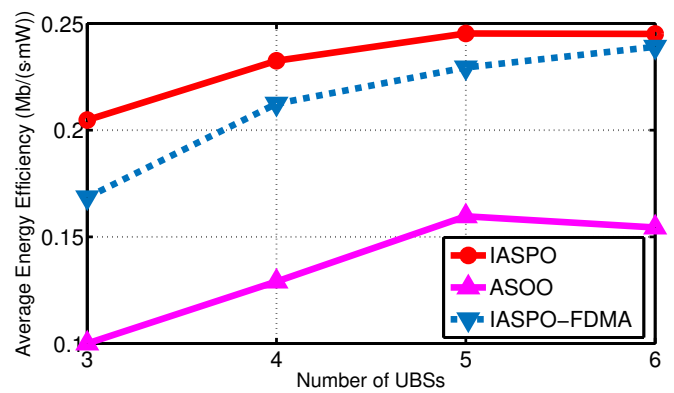

Fig. 3. Average energy efficiency vs. the number of UBSs when $N_{u}=10$.

NOMA affects the resource allocation in our model in two aspects: 1) pros: improve the spectrum efficiency of the network. 2) cons: the association and subchannel optimization can only obtain an approximate solution due to the increased computational complexity. When the number of UEs is great, the exploitation of the NOMA technique improves the spectrum efficiency. However, when the number of UEs is small, the loss of exploiting the NOMA technique is greater than the benefit.

- The energy efficiency of the ASOO and IASPO-FDMA algorithms generally decreases with the increase of the number of UEs, while the energy efficiency of the IASPO algorithm is relatively robust to the increase of the number of UEs.

- The energy efficiency of all comparison algorithms generally increases with the increasing number UBS. However, the deployment of more UBSs means the consumption of more UAV resources. In summary, the above results indicate that our proposed IASPO algorithm can improve the energy efficiency, especially in the scenario where UAV resources are relatively scarce, that is, there are many UEs or few UBSs.

\section{CONCLUSion}

This paper investigated the non-orthogonal resource allocation problem in a multi-UAV-aided network for providing eMBB services and formulated a joint non-orthogonal user association, subchannel allocation and power control problem to maximize the network energy efficiency. To alleviate this problem, we first decomposed it into two separated subproblems, namely, a user association and subchannel allocation subproblem and a power control subproblem. We then designed a two-stage approximation strategy to solve the user association and subchannel allocation subproblem and exploited an SCA approach to approximate the power control subproblem. Based on the above results, we then developed an iterative algorithm with provable convergence to solve the joint optimization problem. Simulation results verified that our proposed algorithm could improve the energy efficiency compared with several benchmark algorithms. This paper assumes that each subchannel $\mathcal{S C}_{j n}$ can be assigned to at most two users, developing some low-complexity algorithms considering more-user NOMA may be a topic worthy of research in the near future.

\section{APPENDIX}

\section{A. Proof of Lemma 2}

Proof. Let $\mathcal{P}^{(r)}=\left\{p_{1, j n}^{(r)}, p_{2, j n}^{(r)}\right\}$ denote the given transmit power point in the $(r+1)$-th iteration $(r \geq 0)$. Next, we discuss how to transform (32) into a convex optimization problem via the SCA approach in detail. Note that we need to approximate the left-hand side of (32 b) as a concave function and the lefthand side of (32) as a convex function.

First, we study the approximation of $r_{i j n}^{p}(\mathcal{P})$ and $r_{i j n}^{s}(\mathcal{P})$. For $r_{i j n}^{p}(\mathcal{P})$, it can be written as a difference of two concave functions with respect to $\mathcal{P}$, i.e.,

$$
r_{i j n}^{p}(\mathcal{P})=\widehat{r}_{i j n}^{p}(\mathcal{P})-\breve{r}_{i j n}^{p}(\mathcal{P}),
$$

where

$$
\begin{aligned}
& \widehat{r}_{i j n}^{p}(\mathcal{P})= \\
& \frac{W}{N_{s}} \log _{2}\left(p_{1, j n} h_{i j n}+\sum_{k \neq j, k \in \mathcal{J}}\left(p_{1, k n}+p_{2, k n}\right) h_{i k n}+p_{n}^{M} h_{i n}^{M}+\sigma_{n}^{2}\right), \\
& \breve{r}_{i j n}^{p}(\mathcal{P})=\frac{W}{N_{s}} \log _{2}\left(\sum_{k \neq j, k \in \mathcal{J}}\left(p_{1, k n}+p_{2, k n}\right) h_{i k n}+p_{n}^{M} h_{i n}^{M}+\sigma_{n}^{2}\right) .
\end{aligned}
$$

It can be proved that any concave function is globally upperbounded by its first-order Taylor expansion at any point [14]. Therefore, we have the following upper bounds of $\widehat{r}_{i j n}^{p}(\mathcal{P})$ and $\breve{r}_{i j n}^{p}(\mathcal{P})$ at the given transmit power point $\mathcal{P}^{(r)}$

$$
\begin{aligned}
& \widehat{r}_{i j n}^{p}(\mathcal{P}) \leq B_{i j n}^{(r)}+D_{i j n}^{(r)}\left(h_{i j n}\left(p_{1, j n}-p_{1, j n}^{(r)}\right)+\right. \\
& \left.\sum_{k \neq j, k \in \mathcal{J}} h_{i k n}\left(p_{1, k n}+p_{2, k n}-p_{1, k n}^{(r)}-p_{2, k n}^{(r)}\right)\right) \\
& =\widehat{r}_{i j n}^{p, t(r)}(\mathcal{P}), \\
& \widehat{r}_{i j n}^{p}(\mathcal{P}) \leq E_{i j n}^{(r)}+ \\
& F_{i j n}^{(r)}\left(\sum_{k \neq j, k \in \mathcal{J}} h_{i k n}\left(p_{1, k n}+p_{2, k n}-p_{1, k n}^{(r)}-p_{2, k n}^{(r)}\right)\right) \\
& =\breve{r}_{i j n}^{p, t(r)}(\mathcal{P}),
\end{aligned}
$$

where

$$
\begin{aligned}
& B_{i j n}^{(r)}=\frac{W}{N_{s}} \log _{2}\left(p_{1, j n}^{(r)} h_{i j n}+\right. \\
& \left.\sum_{k \neq j, k \in \mathcal{J}}\left(p_{1, k n}^{(r)}+p_{2, k n}^{(r)}\right) h_{i k n}+p_{n}^{M} h_{i n}^{M}+\sigma_{n}^{2}\right),
\end{aligned}
$$




$$
\begin{gathered}
D_{i j n}^{(r)}=\frac{\log _{2}(e) W / N_{s}}{p_{1, j n}^{(r)} h_{i j n}+\sum_{k \neq j, k \in \mathcal{J}}^{(r), k n}\left(p_{2, k n}^{(r)}\right) h_{i k n}+p_{n}^{M} h_{i n}^{M}+\sigma_{n}^{2}}, \\
E_{i j n}^{(r)}= \\
\frac{W}{N_{s}} \log _{2}\left(\sum_{k \neq j, k \in \mathcal{J}}\left(p_{1, k n}^{(r)}+p_{2, k n}^{(r)}\right) h_{i k n}+p_{n}^{M} h_{i n}^{M}+\sigma_{n}^{2}\right), \\
F_{i j n}^{(r)}=\frac{\log _{2}(e) W / N_{s}}{\sum_{k \neq j, k \in \mathcal{J}}\left(p_{1, k n}^{(r)}+p_{2, k n}^{(r)}\right) h_{i k n}+p_{n}^{M} h_{i n}^{M}+\sigma_{n}^{2}} .
\end{gathered}
$$

Similarly, $r_{i j n}^{s}(\mathcal{P})$ can be written as a difference of two concave functions with respect to $\mathcal{P}$, i.e.,

$$
r_{i j n}^{s}(\mathcal{P})=\widehat{r}_{i j n}^{s}(\mathcal{P})-\widehat{r}_{i j n}^{s}(\mathcal{P}),
$$

where

$$
\widehat{r}_{i j n}^{s}(\mathcal{P})=\frac{W}{N_{s}} \log _{2}\left(\sum_{k \in \mathcal{J}}\left(p_{1, k n}+p_{2, k n}\right) h_{i k n}+p_{n}^{M} h_{i n}^{M}+\sigma_{n}^{2}\right),
$$$$
\breve{r}_{i j n}^{s}(P)=
$$$$
\frac{W}{N_{s}} \log _{2}\left(p_{1, j n} h_{i j n}+\sum_{k \neq j, k \in \mathcal{J}}\left(p_{1, k n}+p_{2, k n}\right) h_{i k n}+p_{n}^{M} h_{i n}^{M}+\sigma_{n}^{2}\right) .
$$

It can be observed that $\breve{r}_{i j n}^{s}(\mathcal{P})$ and $\widehat{r}_{i j n}^{p}(\mathcal{P})$ have an identical form. Thus, the upper bound of $\breve{r}_{i j n}^{s}(\mathcal{P})$ at $\mathcal{P}^{(r)}$ can be expressed as

$$
\begin{aligned}
& \breve{r}_{i j n}^{s}(\mathcal{P}) \leq B_{i j n}^{(r)}+D_{i j n}^{(r)}\left(h_{i j n}\left(p_{1, j n}-p_{1, j n}^{(r)}\right)+\right. \\
& \left.\sum_{k \neq j, k \in \mathcal{J}} h_{i k n}\left(p_{1, k n}+p_{2, k n}-p_{1, k n}^{(r)}-p_{2, k n}^{(r)}\right)\right) \\
& =\breve{r}_{i j n}^{s, t(r)}(\mathcal{P}) .
\end{aligned}
$$

For $\widehat{r}_{i j n}^{s}(\mathcal{P})$, by leveraging the first-order Taylor expansion, we have the following upper bound of $\widehat{r}_{i j n}^{s}(\mathcal{P})$ at $\mathcal{P}^{(r)}$

$$
\begin{aligned}
& \widehat{r}_{i j n}^{s}(\mathcal{P}) \leq G_{i j n}^{(r)}+ \\
& H_{i j n}^{(r)}\left(\sum_{k \in \mathcal{J}} h_{i k n}\left(p_{1, k n}+p_{2, k n}-p_{1, k n}^{(r)}-p_{2, k n}^{(r)}\right)\right) \\
& =\widehat{r}_{i j n}^{s, t(r)}(\mathcal{P}),
\end{aligned}
$$

where

$$
\begin{gathered}
G_{i j n}^{(r)}=\frac{W}{N_{s}} \log _{2}\left(\sum_{k \in \mathcal{J}}\left(p_{1, k n}^{(r)}+p_{2, k n}^{(r)}\right) h_{i k n}+p_{n}^{M} h_{i n}^{M}+\sigma_{n}^{2}\right), \\
H_{i j n}^{(r)}=\frac{\log _{2}(e) W / N_{s}}{\sum_{k \in \mathcal{J}}\left(p_{1, k n}^{(r)}+p_{2, k n}^{(r)}\right) h_{i k n}+p_{n}^{M} h_{i n}^{M}+\sigma_{n}^{2}}
\end{gathered}
$$

It can be observed that the upper bounds $\widehat{r}_{i j n}^{p, t(r)}(\mathcal{P})$, $\breve{r}_{i j n}^{p, t(r)}(\mathcal{P}), \widehat{r}_{i j n}^{s, t(r)}(\mathcal{P})$, and $\breve{r}_{i j n}^{s, t(r)}(\mathcal{P})$ are linear functions with respect to $\mathcal{P}$.

Next, we study the approximation of the constraints $32 \mathrm{~b}$ ) and (32). By substituting (39) into (35) and substituting (47) into (44), for all $i \in \mathcal{I}$, we can obtain the lower bound of the left-hand side of the constraint (32b) as

$$
\begin{aligned}
& \sum_{(j, n) \in \mathcal{S}_{J N}^{a p}(i)} r_{i j n}^{p}(\mathcal{P})+\sum_{(j, n) \in \mathcal{S}_{J N}^{a s}(i)} r_{i j n}^{s}(\mathcal{P}) \\
\geq & \sum_{(j, n) \in \mathcal{S}_{J N}^{a p}(i)}\left(\widehat{r}_{i j n}^{p}(\mathcal{P})-\widehat{r}_{i j n}^{p, t(r)}(\mathcal{P})\right) \\
+ & \sum_{(j, n) \in \mathcal{S}_{J N}^{a s}(i)}\left(\widehat{r}_{i j n}^{s}(\mathcal{P})-\breve{r}_{i j n}^{s, t(r)}(\mathcal{P})\right) .
\end{aligned}
$$

Similarly, by substituting (38) into (35) and substituting (48) into (44), for all $j \in \mathcal{J}$, we obtain the upper bound of the lefthand side of the constraint (32) as

$$
\begin{aligned}
& \quad \sum_{(i, n) \in \mathcal{S}_{I N}^{a p}(j)} r_{i j n}^{p}(\mathcal{P})+\sum_{(i, n) \in \mathcal{S}_{I N}^{a s}(j)} r_{i j n}^{s}(\mathcal{P}) \\
& \leq \sum_{(i, n) \in \mathcal{S}_{I N}^{a p}(j)}\left(\widehat{r}_{i j n}^{p, t(r)}(\mathcal{P})-\breve{r}_{i j n}^{p}(\mathcal{P})\right) \\
& +\sum_{(i, n) \in \mathcal{S}_{I N}^{a s}(j)}\left(\widehat{r}_{i j n}^{s, t(r)}(\mathcal{P})-\breve{r}_{i j n}^{s}(\mathcal{P})\right) .
\end{aligned}
$$

Therefore, with any given transmit power point $\mathcal{P}^{(r)}=$ $\left\{p_{1, j n}^{(r)}, p_{2, j n}^{(r)}\right\}$, the problem (32) can be approximated as the following form by referring to (51) and (52)

$$
\begin{aligned}
& \max _{\mathcal{P}, \eta_{R}, \eta_{P},\left\{\eta_{i}\right\},\left\{p_{j}\right\}} \eta_{R}-\eta_{E E} \eta_{P} \\
& \text { s.t } \\
& \sum_{(j, n) \in \mathcal{S}_{J N}^{a p}(i)}\left(\widehat{r}_{i j n}^{p}(\mathcal{P})-\breve{r}_{i j n}^{p, t(r)}(\mathcal{P})\right)+ \\
& \sum_{(j, n) \in \mathcal{S}_{J N}^{a s}(i)}\left(\widehat{r}_{i j n}^{s}(\mathcal{P})-\breve{r}_{i j n}^{s, t(r)}(\mathcal{P})\right) \geq \eta_{i}, \forall i \in \mathcal{I}, \\
& \sum_{(i, n) \in \mathcal{S}_{I N}^{a p}(j)}\left(\widehat{r}_{i j n}^{p, t(r)}(\mathcal{P})-\breve{r}_{i j n}^{p}(\mathcal{P})\right)+ \\
& \sum_{(i, n) \in \mathcal{S}_{I N}^{a s}(j)}\left(\widehat{r}_{i j n}^{s, t(r)}(\mathcal{P})-\breve{r}_{i j n}^{s}(\mathcal{P})\right) \leq C_{j}^{\max }, \forall j \in \mathcal{J},
\end{aligned}
$$

(32 $\mathrm{k})$, (32 $\mathrm{d}),(32 \mathrm{f})$, (32 $\mathrm{g}$ ), (32h) and (32 ) are satisfied.

Since the left-hand sides of the constraints $(53 \mathrm{~b})$ and $(53 \mathrm{k})$ are concave and convex with respect to $\mathcal{P}$ respectively, $53 \mathrm{~b}$ ) and (53k) are convex constraints. Therefore, the problem (53) is a convex optimization problem.

Note that the inequalities (51) and (52) indicate that any feasible solution of the problem (53) is also feasible for the problem (32), but the reverse is not true in general. Therefore, the optimal objective value obtained by solving (53) is the lower bound of that of (32).

\section{B. Proof of Lemma 3}

Proof. In the $r$-th iteration $(r \geq 1)$, the obtained $\eta_{E E}\left(\mathcal{A}^{(r)}, \mathcal{P}^{(r)}\right)$ can be expressed as

$$
\eta_{E E}\left(\mathcal{A}^{(r)}, \mathcal{P}^{(r)}\right)=\frac{\eta_{R}\left(\mathcal{A}^{(r)}, \mathcal{P}^{(r)}\right)}{\eta_{P}\left(\mathcal{P}^{(r)}\right)} .
$$

Then in the $(r+1)$-th iteration, after performing the association and subchannel optimization, we can obtain

$$
\eta_{R}\left(\mathcal{A}^{(r+1)}, \mathcal{P}^{(r)}\right) \geq \eta_{R}\left(\mathcal{A}^{(r)}, \mathcal{P}^{(r)}\right) .
$$


According to (54) and (55), $\eta_{E E}\left(\mathcal{A}^{(r+1)}, \mathcal{P}^{(r)}\right)$ satisfies

$$
\begin{aligned}
& \eta_{E E}\left(\mathcal{A}^{(r+1)}, \mathcal{P}(r)\right)=\frac{\eta_{R}\left(\mathcal{A}^{(r+1)}, \mathcal{P}^{(r)}\right)}{\eta_{P}\left(\mathcal{P}^{(r)}\right)} \\
& \geq \frac{\eta_{R}\left(\mathcal{A}^{(r)}, \mathcal{P}^{(r)}\right)}{\eta_{P}\left(\mathcal{P}^{(r)}\right)}=\eta_{E E}\left(\mathcal{A}^{(r)}, \mathcal{P}^{(r)}\right) .
\end{aligned}
$$

After performing the power optimization, we can obtain

$$
\begin{aligned}
& \eta_{R}\left(\mathcal{A}^{(r+1)}, \mathcal{P}^{(r+1)}\right)-\eta_{E E}\left(\mathcal{A}^{(r+1)}, \mathcal{P}^{(r)}\right) \eta_{P}\left(\mathcal{P}^{(r+1)}\right) \\
& \geq \eta_{R}\left(\mathcal{A}^{(r+1)}, \mathcal{P}^{(r)}\right)-\eta_{E E}\left(\mathcal{A}^{(r+1)}, \mathcal{P}^{(r)}\right) \eta_{P}\left(\mathcal{P}^{(r)}\right)=0 .
\end{aligned}
$$

Thus we can obtain

$$
\begin{aligned}
& \eta_{R}\left(\mathcal{A}^{(r+1)}, \mathcal{P}^{(r+1)}\right) \geq \eta_{E E}\left(\mathcal{A}^{(r+1)}, \mathcal{P}^{(r)}\right) \eta_{P}\left(\mathcal{P}^{(r+1)}\right) \\
& \eta_{E E}\left(\mathcal{A}^{(r+1)}, \mathcal{P}^{(r+1)}\right)=\frac{\eta_{R}\left(\mathcal{A}^{(r+1)}, \mathcal{P}^{(r+1)}\right)}{\eta_{P}\left(\mathcal{P}^{(r+1)}\right)} \\
& \geq \eta_{E E}\left(\mathcal{A}^{(r+1)}, \mathcal{P}^{(r)}\right)
\end{aligned}
$$

According to (56) and (59), we can obtain

$$
\eta_{E E}\left(\mathcal{A}^{(r+1)}, \mathcal{P}^{(r+1)}\right) \geq \eta_{E E}\left(\mathcal{A}^{(r)}, \mathcal{P}^{(r)}\right) .
$$

and thus the convergence of the IASPO algorithm is proved.

The complexity of the IASPO algorithm is dominated by that of solving (24), (31) and (53). The complexities of solving the ILP problems (24), (31) are both $O\left(\left(N_{u}+1\right)^{N_{d} \cdot N_{s}}\right)$, and the complexity of solving the convex problem $(53)$ is $O\left(\left(2 N_{d}\right.\right.$. $\left.N_{s}\right)^{3.5}$ ). Moreover, since (24), (31) and (53) need to be iteratively solved until the IASPO algorithm converges or reaches the maximum number of iterations $r_{\max }$, the complexity of the IASPO algorithm is $O\left(r_{\max }\left(2\left(N_{u}+1\right)^{N_{d} \cdot N_{s}}+\left(2 N_{d} \cdot N_{s}\right)^{3.5}\right)\right)$ in the worst case. Although the complexity of the IASPO algorithm is exponential to $N_{d} \cdot N_{s}$, the actual complexity is usually much less than that of the worst case.

\section{REFERENCES}

[1] M. Series, "IMT Vision-framework and overall objectives of the future development of IMT for 2020 and beyond," Recommendation ITU, Tech. Rep. M.2083, 2015.

[2] G. K. Xilouris, M. C. Batistatos, G. E. Athanasiadou, G. Tsoulos, H. B. Pervaiz, and C. C. Zarakovitis, "UAV-assisted 5G network architecture with slicing and virtualization," in 2018 IEEE Globecom Workshops (GC Wkshps). IEEE, 2018, pp. 1-7.

[3] S. Zhang, H. Zhang, B. Di, and L. Song, "Cellular UAV-to-X communications: Design and optimization for multi-UAV networks," IEEE Transactions on Wireless Communications, vol. 18, no. 2, pp. 13461359, 2019.

[4] J. Cui, Y. Liu, and A. Nallanathan, "Multi-agent reinforcement learningbased resource allocation for UAV networks," IEEE Transactions on Wireless Communications, vol. 19, no. 2, pp. 729-743, 2019.

[5] N. Zhao, X. Pang, Z. Li, Y. Chen, F. Li, Z. Ding, and M.-S. Alouini, "Joint trajectory and precoding optimization for UAV-assisted NOMA networks," IEEE Transactions on Communications, vol. 67, no. 5, pp. 3723-3735, 2019.

[6] R. Tang, J. Cheng, and Z. Cao, "Joint placement design, admission control, and power allocation for NOMA-based UAV systems," IEEE Wireless Communications Letters, 2019.

[7] R. Duan, J. Wang, C. Jiang, H. Yao, Y. Ren, and Y. Qian, "Resource allocation for multi-UAV aided IoT NOMA uplink transmission systems," IEEE Internet of Things Journal, vol. 6, no. 4, pp. 7025-7037, 2019.

[8] A. Al-Hourani, S. Kandeepan, and S. Lardner, "Optimal LAP altitude for maximum coverage," IEEE Wireless Communications Letters, vol. 3, no. 6, pp. 569-572, 2014.

[9] T. J. Rouphael, $R F$ and digital signal processing for software-defined radio: a multi-standard multi-mode approach. Newnes, 2009.
[10] H. Zhang, B. Wang, C. Jiang, K. Long, A. Nallanathan, V. C. Leung, and H. V. Poor, "Energy efficient dynamic resource optimization in NOMA system," IEEE Transactions on Wireless Communications, vol. 17, no. 9, pp. 5671-5683, 2018.

[11] Z. Xiao, L. Zhu, J. Choi, P. Xia, and X.-G. Xia, "Joint power allocation and beamforming for non-orthogonal multiple access (NOMA) in 5G millimeter wave communications," IEEE Transactions on Wireless Communications, vol. 17, no. 5, pp. 2961-2974, 2018.

[12] J. Lee and S. Leyffer, Mixed integer nonlinear programming. Springer Science \& Business Media, 2011, vol. 154.

[13] MOSEK ApS, "Introducing the MOSEK optimization suite 8.1.0.82," https://docs.mosek.com/8.1/intro/index.html 2019.

[14] S. Boyd and L. Vandenberghe, Convex optimization. Cambridge university press, 2004. 